\title{
Microinstabilities in the Transition Region of Weak Quasi-perpendicular Intracluster Shocks
}

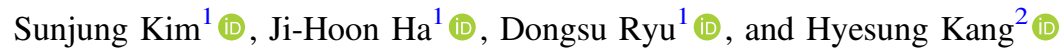 \\ ${ }^{1}$ Department of Physics, School of Natural Sciences, UNIST, Ulsan 44919, Republic of Korea; sunjungkim@ @unist.ac.kr \\ ${ }^{2}$ Department of Earth Sciences, Pusan National University, Busan 46241, Republic of Korea \\ Received 2021 February 15; revised 2021 March 23; accepted 2021 March 23; published 2021 May 21
}

\begin{abstract}
Microinstabilities play important roles in both entropy generation and particle acceleration in collisionless shocks. Recent studies have suggested that in the transition region of quasi-perpendicular $\left(Q_{\perp}\right)$ shocks in the high-beta $\left(\beta=P_{\text {gas }} / P_{\mathrm{B}}\right)$ intracluster medium (ICM), the ion temperature anisotropy due to the reflected-gyrating ions could trigger the Alfvén ion cyclotron (AIC) instability and the ion-mirror instability, while the electron temperature anisotropy induced by magnetic field compression could excite the whistler instability and the electron-mirror instability. Adopting the numerical estimates for ion and electron temperature anisotropies found in the particle-incell (PIC) simulations of $Q_{\perp}$ shocks with sonic Mach numbers, $M_{\mathrm{s}}=2-3$, we carry out a linear stability analysis for these microinstabilities. The kinetic properties of the microinstabilities and the ensuing plasma waves on both ion and electron scales are described for wide ranges of parameters, including $\beta$ and the ion-to-electron mass ratio. In addition, the nonlinear evolution of the induced plasma waves are examined by performing 2D PIC simulations with periodic boundary conditions. We find that for $\beta \approx 20-100$, the AIC instability could induce ion-scale waves and generate shock surface ripples in supercritical shocks above the AIC critical Mach number, $M_{\mathrm{AIC}}^{*} \approx 2.3$. Also, electron-scale waves are generated primarily by the whistler instability in these high- $\beta$ shocks. The resulting multiscale waves from electron to ion scales are thought to be essential in the electron injection to diffusive shock acceleration in $Q_{\perp}$ shocks in the ICM.
\end{abstract}

Unified Astronomy Thesaurus concepts: Shocks (2086); Plasma astrophysics (1261)

\section{Introduction}

Major mergers of galaxy clusters are known to drive weak shocks with sonic Mach numbers, $M_{\mathrm{s}} \lesssim 3$, in the hot intracluster medium (ICM) of high $\beta$ (e.g., Ryu et al. 2003; Skillman et al. 2008; Vazza et al. 2009; Hong et al. 2014; Ha et al. 2018). Here, the plasma beta, $\beta=P_{\text {gas }} / P_{\mathrm{B}}$, is the ratio of the gas pressure to the magnetic pressure. The radiative signatures of such shocks have been detected in X-ray and radio observations (e.g., Brüggen et al. 2012; Brunetti \& Jones 2014). In the case of so-called radio relics, the radio emission has been interpreted as the synchrotron radiation from relativistic electrons accelerated via diffusive shock acceleration (DSA) in merger-driven shocks (see van Weeren et al. 2019 for a review).

To explain the origin of radio relics, this DSA model requires an electron preacceleration mechanism because postshock thermal electrons do not have momenta large enough to participate in the standard DSA process, in which cosmic-ray (CR) electrons diffuse across the shock (Kang et al. 2012). Because the width of the shock transition layer is comparable to the gyroradius of postshock thermal ions, thermal electrons need to be energized to the so-called injection momentum, $p_{\text {inj }} \sim \mathrm{a}$ few $\times_{p i, \text { th }}$. Here, $p_{\mathrm{i}, \text { th }}=\sqrt{2 m_{\mathrm{i}} k_{\mathrm{B}} T_{\mathrm{i} 2}}$ is the ion thermal momentum in the postshock gas of temperature $T_{\mathrm{i} 2}, m_{i}$ is the ion mass, and $k_{\mathrm{B}}$ is the Boltzmann constant. For shocks in the solar wind, the electron injection is observed preferentially at the quasi-perpendicular $\left(Q_{\perp}\right)$ configuration with $\theta_{\mathrm{Bn}} \gtrsim 45^{\circ}$, where $\theta_{\mathrm{Bn}}$ is the shock obliquity angle between the shock normal and the upstream magnetic field

Original content from this work may be used under the terms of the Creative Commons Attribution 4.0 licence. Any further distribution of this work must maintain attribution to the author(s) and the title of the work, journal citation and DOI. direction (e.g., Gosling et al. 1989; Burgess 2006; Oka et al. 2006).

The electron preacceleration has been a key outstanding problem in understanding the production of $\mathrm{CR}$ electrons in weak ICM shocks. Previous studies have shown that, in low- $M_{\mathrm{s}}$, high- $\beta \quad Q_{\perp}$ shocks, thermal electrons could be preaccelerated primarily through the Fermi-like acceleration in the shock foot (Matsukiyo et al. 2011; Guo et al. 2014a, 2014b; Kang et al. 2019) and the stochastic shock drift acceleration (SSDA) in the shock transition region (Katou \& Amano 2019; Niemiec et al. 2019; Trotta \& Burgess 2019). Although it has been shown that the electron preacceleration would be enhanced by preexisting strong magnetic fluctuations in the low- $\beta(\sim 1)$ regime (e.g., Guo \& Giacalone 2015; Trotta et al. 2020), the effect of such turbulence on high- $\beta$ ICM shocks has yet to be investigated and will not be considered here.

Both the Fermi-like acceleration and SSDA mechanisms rely on the various microinstabilities triggered by the ion and electron temperature anisotropies in the shock structure (Gary 1993). If $T_{\mathrm{e} \|}>T_{\mathrm{e} \perp}$, for example, the electron firehose instability (EFI) can grow with the following two branches: the nonresonant, parallelpropagating mode with left-hand circular polarization (LHCP) and the resonant, nonpropagating, oblique mode (Gary \& Nishimura 2003). Hereafter, the subscripts $\|$ and $\perp$ denote the parallel and perpendicular directions to the background magnetic field, $\boldsymbol{B}_{0}$, respectively. Under the condition of $T_{\mathrm{e} \perp}>T_{\mathrm{e} \|}$, by contrast, the whistler instability and the electron-mirror (e-mirror) instability can be triggered (Scharer \& Trivelpiece 1967; Gary 1992; Hellinger \& Štverák 2018). The most unstable whistler mode propagates in the direction parallel to $\boldsymbol{B}_{0}$ with right-hand circular polarization (RHCP), while the e-mirror mode is nonpropagating and has the maximum growth rate at the wavevector direction oblique with respect to $\boldsymbol{B}_{0}$. In the case of $T_{\mathrm{i} \perp}>T_{\mathrm{i} \|}$, the Alfvén ion 
Table 1

Linear Properties of the Instabilities Driven by Perpendicular Temperature Anisotropies

\begin{tabular}{|c|c|c|c|c|}
\hline Instability & AIC & Whistler & Ion-mirror & Electron-mirror \\
\hline Free energy source & $T_{\mathrm{i} \perp}>T_{\mathrm{i} \|}$ & $T_{\mathrm{e} \perp}>T_{\mathrm{e} \|}$ & $T_{\mathrm{i} \perp}>T_{\mathrm{i} \|}$ & $T_{\mathrm{e} \perp}>T_{\mathrm{e} \|}$ \\
\hline Propagation angle with $\gamma_{m}{ }^{\mathrm{a}}$ & parallel & parallel & oblique & oblique \\
\hline Wavenumber & $c k / \omega_{\mathrm{pi}} \leqslant 1$ & $c k / \omega_{\mathrm{pe}} \leqslant 1$ & $c k / \omega_{\mathrm{pi}} \leqslant 1$ & $c k / \omega_{\mathrm{pe}} \leqslant 1$ \\
\hline Wave frequency & $0<\omega_{r}<\Omega_{\mathrm{ci}}$ & $\Omega_{\mathrm{ci}}<\omega_{r}<\Omega_{\mathrm{ce}}$ & $\omega_{r}=0$ & $\omega_{r}=0$ \\
\hline Wave polarization & $\mathrm{LHCP}^{\mathrm{b}}$ & $\mathrm{RHCP}^{\mathrm{b}}$ & Nonpropagating & Nonpropagating \\
\hline
\end{tabular}

Notes.

${ }^{\text {a }} \gamma_{m}$ is the maximum growth rate.

${ }^{\mathrm{b}}$ LHCP (RHCP) stands for left-hand (right-hand) circular polarization.

cyclotron instability (AIC, or the proton cyclotron instability) and the ion-mirror (i-mirror) instability may become unstable (Winske \& Quest 1988; Gary 1993; Gary et al. 1997; Burgess 2006). The fastest-growing mode of the AIC instability propagates in the direction parallel to $\boldsymbol{B}_{0}$ with LHCP, while the i-mirror mode is nonpropagating and has the maximum growth rate at the wavevector direction oblique with respect to $\boldsymbol{B}_{0}$. Table 1 summarizes these linear properties of the instabilities driven by perpendicular temperature anisotropies, which are relevant for the present study.

In the foot of $Q_{\perp}$ shocks, the shock-reflected electrons backstream mainly along the upstream magnetic field and induce an electron parallel anisotropy $\left(T_{\mathrm{e} \|}>T_{\mathrm{e} \perp}\right)$, which could trigger the EFI and facilitate the Fermi-like preacceleration (Guo et al. 2014b; Kang et al. 2019; Kim et al. 2020). In the transition region behind the shock ramp, on the other hand, the AIC and i-mirror instabilities can be triggered by the ion perpendicular anisotropy $\left(T_{\mathrm{i} \perp}>T_{\mathrm{i} \|}\right)$ mainly due to the shock-reflected ions advected downstream, while the whistler and e-mirror instabilities can be excited by the electron perpendicular anisotropy $\left(T_{\mathrm{e} \perp}>T_{\mathrm{e} \|}\right)$ mainly due to magnetic field compression at the shock ramp (Guo et al. 2017; Katou \& Amano 2019). Such multiscale waves from electron to ion scales are essential in the electron preacceleration via the SSDA (Matsukiyo \& Matsumoto 2015; Niemiec et al. 2019; Trotta \& Burgess 2019).

Using two-dimensional (2D) particle-in-cell (PIC) simulations for $\beta \approx 20-100 Q_{\perp}$ shocks, Kang et al. (2019) showed that the Fermi-like preacceleration involving multiple cycles of shock drift acceleration (SDA) in the shock foot could be effective only in supercritical shocks with $M_{\mathrm{s}}$ greater than the EFI critical Mach number, $M_{\mathrm{ef}}^{*} \approx 2.3$. However, they argued that the electron preacceleration may not proceed all the way to $p_{\text {inj }}$ because the EFI-driven waves are limited to electron scales. Niemiec et al. (2019), on the other hand, performed a PIC simulation for $M_{\mathrm{s}}=3$ shock with $\beta=5$ in a 2D domain large enough to include ion-scale fluctuations and suggested that electrons could be energized beyond $p_{\text {inj }}$ via the SSDA due to stochastic pitch-angle scattering off the multiscale waves excited in the shock transition zone.

Furthermore, Trotta \& Burgess (2019) found that in $\beta \approx 1$ plasmas, the AIC instability is triggered, and the ensuing electron preacceleration operates only in supercritical shocks with Alfvénic Mach number greater than the critical Mach number, $M_{\mathrm{AIC}}^{*} \approx 3.5$. In a separate paper (Ha et al. 2021, HKRK2021 hereafter), we report a similar study of $\beta \approx 20-100$ shocks, which is design to explore through 2D PIC simulations how the multiscale waves excited mainly by the AIC and whistler instabilities in the shock transition can assist the electron injection to DSA in ICM shocks.
In this paper, adopting the numerical estimates for the temperature anisotropies in the transition region of the simulated shocks of HKRK $2021,{ }^{3}$ we first perform a linear stability analysis for microinstabilities for wide ranges of parameters such as $M_{\mathrm{s}}=2-3, \beta=1-100$, and the ion-toelectron mass ratio $m_{i} / m_{e}=50-1836$. This approach allows us to identify the most dominant modes of possible microinstabilities and to evaluate their linear properties for the set of realistic parameters pertaining to ICM shocks. Hence, this kind of analysis on kinetic scales can provide crucial insights for theoretical modelings and/or larger-scale simulations of particle acceleration at weak high- $\beta$ shocks. However, one of the limitations of PIC simulations is that they can follow kinetic plasma processes mainly at the low end of temporal and spatial scales owing to severe requirements of computational resources (e.g., Pohl et al. 2020).

In addition, adopting the same setup as in the linear analysis but only for the models with $\beta=50$ and $m_{i} / m_{e}=50$, we carry out 2D PIC simulations with periodic boundary conditions (periodic-box simulations hereafter) to study the nonlinear evolution of the plasma waves excited by such microinstabilities. Note that throughout the paper. we refer to two different sets of PIC simulations: (1) the "periodic-box simulations" are designed to study the nonlinear evolution of the excited plasma waves in the same setup as in the linear analysis and will be presented in Section 3. (2) The "shock simulations" reported in HKRK2021 provide the numerical estimates for the ion and electron temperature anisotropies in the shock transition zone.

The paper is organized as follows. Section 2 describes the linear analysis of the AIC, whistler, and mirror instabilities. In Section 3, we present the evolution of the waves driven by these instabilities in 2D periodic-box simulations. In Section 4, the implication of our work for the shock criticality and shock surface ripples is discussed. A brief summary is given in Section 5.

\section{Linear Analysis \\ 2.1. Basic Equations}

We consider a homogeneous, collisionless, magnetized plasma, which is specified by the density and temperature of ions and electrons, $n_{\mathrm{i}}, n_{\mathrm{e}}, T_{\mathrm{i}}, T_{\mathrm{e}}$, and the background magnetic field $\boldsymbol{B}_{0}$. The linear dispersion relation of general

\footnotetext{
3 In HKRK2021 and hereafter, the transition zone is defined as the downstream region of $r_{\mathrm{L}, \mathrm{i}}$ behind the shock ramp, where $r_{\mathrm{L}, \mathrm{i}} \approx u_{0} / \Omega_{\mathrm{ci}}^{\text {up }}$ is the gyroradius of incoming ions; $u_{0}$ is the preshock flow speed defined in the downstream rest frame, and $\Omega_{\text {ci }}^{\text {up }}$ is the gyrofrequency in the upstream. Both the first and second overshoots and the accompanying undershoot are included in this zone, beyond which the downstream states satisfy the canonical RankineHugoniot relation (see Figure 1 of HKRK2021).
} 
(a)

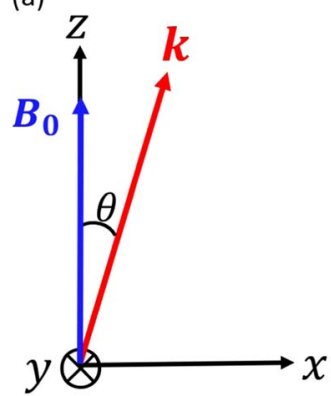

(b)

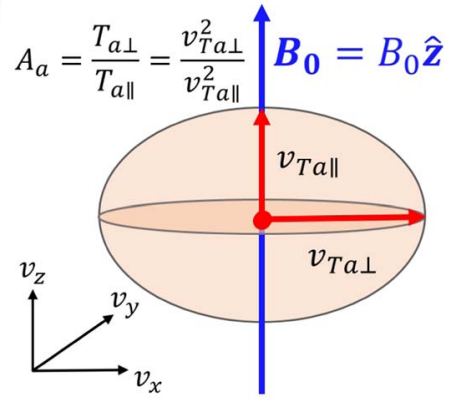

Figure 1. (a) Coordinate system employed in the present study. The background magnetic field, $\boldsymbol{B}_{0}=B_{0} \hat{z}$, is parallel to the $+\hat{z}$ direction, while the wavevector, $\boldsymbol{k}=k_{x} \hat{x}+k_{z} \hat{z}$, lies in the $x-z$ plane. The wave propagation angle, $\theta$, is the angle between $\boldsymbol{B}_{0}$ and $\boldsymbol{k}$. (b) Schematic configuration showing the velocity ellipsoid of a bi-Maxwellian VDF with a temperature anisotropy $\mathcal{A}_{a}$, where $a$ denotes either "ion" or "electron."

electromagnetic (EM) modes is given as

$$
\operatorname{det}\left(\epsilon_{i j}-\frac{c^{2} k^{2}}{\omega^{2}}\left(\delta_{i j}-\frac{k_{i} k_{j}}{k^{2}}\right)\right)=0,
$$

where the dielectric tensor, $\epsilon_{i j}$, is determined by the plasma parameters and the velocity distribution functions (VDFs) of particles. Here, $k_{i}$ and $k_{j}$ are the components of the wavevector $\boldsymbol{k}$. Then, the complex frequency, $\omega=\omega_{r}+i \gamma,{ }^{4}$ can be calculated as a function of the wavenumber, $k$, and the propagating angle, $\theta$, between $\boldsymbol{k}$ and $\boldsymbol{B}_{0}$. Without loss of generality, we set $\boldsymbol{B}_{0}=B_{0} \hat{z}$ along the $+z$ direction and $\boldsymbol{k}=k_{x} \hat{x}+k_{z} \hat{z}$ in the $x-z$ plane, as schematically illustrated in Figure 1(a).

In order to compute $\epsilon_{i j}$, we adopt the VDFs with biMaxwellian distributions for ions and electrons:

$$
f_{a}\left(v_{\perp}, v_{\|}\right)=\frac{n_{0}}{\pi^{3 / 2} v_{T a \perp}^{2} v_{T a \|}} \exp \left(-\frac{v_{\perp}^{2}}{v_{T a \perp}^{2}}-\frac{v_{\|}^{2}}{v_{T a \|}^{2}}\right),
$$

where $v_{\perp}=\sqrt{v_{x}^{2}+v_{y}^{2}}$ and $v_{\|}=v_{z}$. The subscript $a$ denotes $e$ or $i$ defined as the electron or ion species, respectively. Here, $n_{0}$ is the number density of electrons or ions, which satisfies the charge neutrality condition, i.e., $n_{0}=n_{e}=n_{i}$. The parallel and perpendicular (to $\boldsymbol{B}_{0}$ ) thermal velocities are $v_{T a \|}=\sqrt{2 k_{\mathrm{B}} T_{a \|} / m_{a}}$ and $v_{T a \perp}=\sqrt{2 k_{B} T_{a \perp} / m_{a}}$, respectively. Then, the perpendicular temperature anisotropy of each particle species is given as $\mathcal{A}_{a} \equiv T_{a \perp} / T_{a \|}=v_{T a \perp}^{2} / v_{T a \|}^{2}$. The schematic configuration of the thermal velocity ellipsoid for a bi-Maxwellian VDF with the temperature anisotropy $\mathcal{A}_{a}$ is shown in Figure 1(b). As $\mathcal{A}_{a}$ increases, the thermal velocity surface in the velocity space deviates further away from the spherical shape. Under these considerations, $\epsilon_{i j}$ is given as Equation (3) in Kim et al. (2020) without the bulk drift velocities. We note that in the shock simulations of HKRK2021, the VDFs of ions and electrons in the transition zone are likely nongyrotropic and non-Maxwellian due to the SDA-reflected ions and electrons accelerated via the gradient- $B$ drift (see Figure 4 of Guo et al. 2014a). However, we expect the effects of non-Maxwellian VDFs on the linear predictions would be only marginal because the fractions of

\footnotetext{
4 The quantity $i$ is the imaginary unit, and not the coordinate component nor ion species.
}

particles in the suprathermal tail are on order of $10^{-2}$ for ions and $\ll 10^{-2}$ for electrons in the downstream region of the fiducial $M_{\mathrm{s}}=3$ shock model (Kang et al. 2019).

For $n_{0}, \boldsymbol{B}_{0}, T_{a \|}$, and $T_{a \perp}$ of the homogeneous background plasma, we adopt the numerical values, averaged over the transition zone of the simulated shocks of HKRK2021, where the preshock conditions are specified with the typical parameters of high- $\beta$ ICM plasmas, $n_{\mathrm{ICM}}=10^{-4} \mathrm{~cm}^{-3}, k_{\mathrm{B}} T_{\mathrm{ICM}}=\left(k_{\mathrm{B}} T_{\mathrm{i}}+\right.$ $\left.k_{\mathrm{B}} T_{\mathrm{e}}\right) / 2=8.6 \mathrm{KeV}$, and $\beta_{\mathrm{ICM}}=20-100$. Again, in the shock simulations, both the ion and electron distributions are spatially nonuniform in the transition zone, where the flow structure oscillates with overshoots and undershoots in the longitudinal direction and ripples in the transverse direction. Hence, we focus on qualitative analyses of the instabilities rather than making precise quantitative predictions.

Throughout the paper, the plasma beta, $\beta_{a}=8 \pi n_{a} k_{B} T_{a} / B_{0}^{2}$, the plasma frequency, $\omega_{\mathrm{pa}}^{2}=4 \pi n_{a} e^{2} / m_{a}$, and the gyrofrequency, $\Omega_{\mathrm{ca}}=e B_{0} / m_{a} c$, for electrons and ions are used. Note that in HKRK2021 the results are expressed in terms of the upstream parameters, $n_{0}^{\text {up }} \approx n_{0} / r$ and $B_{0}^{\text {up }} \approx B_{0} / r$, where $r$ is the density compression ratio across the shock ramp. So, for example, $\omega_{\mathrm{pa}}^{\mathrm{up}} \approx \omega_{\mathrm{pa}} / \sqrt{r}$ and $\Omega_{\mathrm{ca}}^{\mathrm{up}} \approx \Omega_{\mathrm{ca}} / r$.

Plasma waves are characterized with the growth rate, $\gamma$, and the real frequency, $\omega_{r}$, which are calculated by solving the dispersion relation in Equation (1) for wavevector $\boldsymbol{k}$. If the propagation angle of the wave with the maximum growth rate, $\gamma_{m}$, is $\theta_{m} \approx 0^{\circ}$, the wave mode is called "parallel-propagating." If $\theta_{m} \gg 0^{\circ}$, it is "oblique-propagating." If the wave frequency, $\omega_{r} \approx 0$, the mode is "nonpropagating." The wave polarization, $P$, can also be estimated using the solution of the dispersion relation as follows:

$$
P \equiv \operatorname{sign}\left(\omega_{r}\right) \frac{\left|\delta E_{+}\right|-\left|\delta E_{-}\right|}{\left|\delta E_{+}\right|+\left|\delta E_{-}\right|},
$$

where $\delta E_{ \pm} \equiv \delta E_{\boldsymbol{k}, \omega}^{x} \mp i \delta E_{\boldsymbol{k}, \omega}^{y}$ (Verscharen \& Chandran 2013). The LHCP corresponds to $P=-1$, whereas the RHCP corresponds to $P=+1$. Waves are in general elliptically polarized with $P \neq \pm 1$. In the case of the nonpropagating mode $\left(\omega_{r}=0\right), P=0$ (see Table 1$)$.

\subsection{Linear Properties of AIC, Whistler, and Mirror Instabilities}

In this section, we report the results of the linear stability analysis for the microinstabilities triggered by the ion and electron temperature anisotropies in the transition region of high- $\beta Q_{\perp}$ shocks. The first column of Table 2 lists the model name, which is assigned with the two parameters, the shock Mach number, $M_{\mathrm{s}}$, and $\beta\left(\approx \beta_{\mathrm{e}}+\beta_{\mathrm{i}}\right)$ in the shock transition region. For example, the LM3.0 $\beta 50$ model has $M_{\mathrm{s}}=3.0$ and $\beta \approx 50$. The values of the parameters, $\beta_{\mathrm{e}}, \beta_{\mathrm{i}}, \mathcal{A}_{\mathrm{e}}$, and $\mathcal{A}_{\mathrm{i}}$, are listed in columns 3-6 of the table. For the models of $\beta \approx 20-100$ and the mass ratio $m_{i} / m_{e}=50$, they are obtained with $n_{0}, \boldsymbol{B}_{0}, T_{a \|}$, and $T_{a \perp}$ estimated by averaging the numerical values over the transition region in the simulated shocks with $M_{\mathrm{s}}=2-3$ and $\beta^{\text {up }}=20-100$ of HKRK2021..$^{5}$ Considering the uncertainties in averaging over nonlinear structures with

\footnotetext{
5 Note that $\beta^{\text {up }}$ for the shock models in HKRK2021 represents the plasma beta of the upstream, preshock plasmas, while $\beta$ for the linear analysis models in Table 2 is the plasma beta in the shock transition zone. We found that $\beta \approx \beta^{\text {up }}$ for the simulated shocks, although, in general, the plasma beta of the far downstream region is higher than $\beta^{\mathrm{up}}$.
} 
Table 2

Model Parameters and Linear Predictions

\begin{tabular}{|c|c|c|c|c|c|c|c|c|c|c|}
\hline Model Name & $M_{\mathrm{s}}$ & $\beta_{\mathrm{e}}^{\mathrm{a}}$ & $\beta_{\mathrm{i}}^{\mathrm{a}}$ & $\mathcal{A}_{\mathrm{e}}^{\mathrm{a}}$ & $\mathcal{A}_{\mathrm{i}}^{\mathrm{a}}$ & $m_{\mathrm{i}} / m_{\mathrm{e}}$ & $\mathrm{AIC}^{\mathrm{b}}$ & Whistler $^{\mathrm{c}}$ & Ion-mirror $^{\mathrm{b}}$ & Electron-mirror ${ }^{c}$ \\
\hline LM2.0 $\beta 20$ & 2.0 & 9.7 & 10.3 & 1.1 & 1.1 & 50 & stable & stable & stable & stable \\
\hline LM2.0 $\beta 50$ & 2.0 & 24 & 26 & 1.1 & 1.2 & 50 & stable & $(0.013,0.20,0)$ & quasi-stable & $(0.0029,0.14,69)$ \\
\hline LM2.0 $\beta 100$ & 2.0 & 48 & 52 & 1.1 & 1.2 & 50 & quasi-stable & $(0.035,0.20,0)$ & $(0.015,0.14,62)$ & $(0.008,0.15,56)$ \\
\hline LM2.3 $\beta 20$ & 2.3 & 8.4 & 12 & 1.1 & 1.5 & 50 & $(0.041,0.21,0)$ & $(0.0016,0.26,0)$ & $(0.04,0.28,63)$ & stable \\
\hline LM2.3 $\beta 50$ & 2.3 & 22 & 28 & 1.2 & 1.5 & 50 & $(0.048,0.15,0)$ & $(0.03,0.24,0)$ & $(0.054,0.21,61)$ & $(0.0074,0.18,67)$ \\
\hline LM2.3 $\beta 100$ & 2.3 & 44 & 56 & 1.2 & 1.5 & 50 & $(0.053,0.11,0)$ & $(0.056,0.24,0)$ & $(0.063,0.16,58)$ & $(0.016,0.17,61)$ \\
\hline LM3.0 $\beta 20$ & 3.0 & 7.5 & 13 & 1.2 & 2.0 & 50 & $(0.127,0.29,0)$ & $(0.0156,0.30,0)$ & $(0.094,0.32,56)$ & $(0.0016,0.17,74)$ \\
\hline LM3.0 $\beta 50$ & 3.0 & 19 & 31 & 1.2 & 2.0 & 50 & $(0.145,0.20,0)$ & $(0.059,0.29,0)$ & $(0.11,0.23,55)$ & $(0.015,0.22,64)$ \\
\hline LM3.0 $\beta 100$ & 3.0 & 38 & 62 & 1.2 & 2.0 & 50 & $(0.156,0.15,0)$ & $(0.10,0.29,0)$ & $(0.12,0.18,54)$ & $(0.03,0.21,56)$ \\
\hline LM3.0 $\beta 50-\mathrm{m} 100$ & 3.0 & 19 & 31 & 1.2 & 2.0 & 100 & $(0.145,0.20,0)$ & $(0.065,0.29,0)$ & $(0.11,0.23,55)$ & $(0.016,0.22,64)$ \\
\hline LM3.0 $\beta 50-\mathrm{m} 1836$ & 3.0 & 19 & 31 & 1.2 & 2.0 & 1836 & $(0.145,0.20,0)$ & $(0.072,0.29,0)$ & $(0.12,0.24,55)$ & $(0.016,0.22,64)$ \\
\hline
\end{tabular}

Notes.

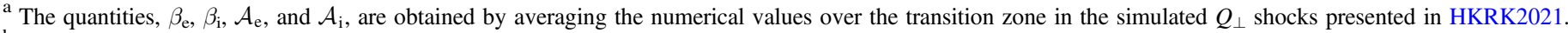

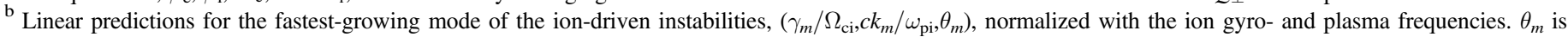
given in units of degree.

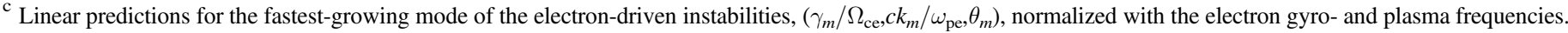
$\theta_{m}$ is given in units of degrees.

overshoot/undershoot oscillations, they are given only up to two significant figures.

For the models with higher mass ratios, LM3.0 $350-\mathrm{m} 100$ with $m_{i} / m_{e}=100$ and LM3.0 $350-\mathrm{m} 1836$ with $m_{i} / m_{e}=1836$, the parameters for the LM3.0 $\beta 50 \operatorname{model}\left(\beta_{\mathrm{e}}=19, \beta_{\mathrm{i}}=31, \mathcal{A}_{\mathrm{e}}=1.2\right.$, and $\mathcal{A}_{\mathrm{i}}=2.0$ ) are used only for the linear analysis. Also, we carried out two additional shock simulations for M3.0 $\beta 1$ with $\beta=1$ and M3.0 $\beta 5$ with $\beta=5$, which were not considered in HKRK2021, in order to obtain the parameters to be used for LM3.0 $\beta 1$ and LM3.0 35 . Our fiducial models have $m_{i} / m_{e}=50$, which is adopted in order to ease the requirements of computational resources for the periodic-box PIC simulations that will be described in Section 3 .

The linear predictions for the AIC, whistler, i-mirror, and e-mirror instabilities are given in columns $8-11$ of Table 2 . The three numbers inside the parentheses present the linear properties of the fastest-growing mode: $\left(\gamma_{m} / \Omega_{\mathrm{ci}}, c k_{m} / \omega_{\mathrm{pi}}, \theta_{m}\right)$ for the AIC and i-mirror instabilities, and $\left(\gamma_{m} / \Omega_{\mathrm{ce}}, c k_{m} / \omega_{\mathrm{pe}}, \theta_{m}\right)$ for the whistler and e-mirror instabilities. Here, $k_{m}$ is the wavenumber that has the maximum growth rate $\gamma_{m}$ at $\theta_{m}$, and $\theta_{m}$ is given in units of degrees. For a clear distinction between the ion- and electronmirror modes, in columns 10-11, the $\gamma_{m}$ of each mirror mode, obtained with either isotropic electrons (i.e., $\mathcal{A}_{\mathrm{e}}=1, \mathcal{A}_{\mathrm{i}}>1$ ) or isotropic ions (i.e., $\mathcal{A}_{\mathrm{i}}=1, \mathcal{A}_{\mathrm{e}}>1$ ), is shown. Note that "stable" means that waves cannot grow because $\gamma_{m}<0$, and "quasi-stable" corresponds to $\gamma_{m} / \Omega_{\mathrm{ci}}<10^{-2}$.

Figure 2 shows the linear analysis results for the LM3.0 $\beta 50$ m1836 model. For the adopted parameters, $\omega_{\mathrm{pe}} / \Omega_{\mathrm{ce}}=26$. Panels (a)-(c) display the growth (or damping) rate at $\theta_{m}$ of the AIC, whistler, and mirror instabilities, respectively, as a function of the wavenumber. To make a uniform comparison, $\gamma$ and $k$ are normalized with $\Omega_{\mathrm{ci}}$ and $\omega_{\mathrm{pi}} / c$, respectively, for both the ion-driven and electron-driven instabilities. Note that in panel (c) both $\gamma$ and $k$ are given in logarithmic scales in order to show both the i-mirror and e-mirror modes in the same panel. To examine the effects of $\mathcal{A}_{\mathrm{i}}$ and $\mathcal{A}_{\mathrm{e}}$ separately and also their combination, we present the black dashed lines for the case with both the ion and electron anisotropies, the red solid lines with the ion anisotropy only, and the blue solid lines with the electron anisotropy only.

The AIC instability induces quasi-parallel modes with $\theta_{m}=0^{\circ}$. Although $\mathcal{A}_{\mathrm{i}}>1$ is the main free energy source that drives the AIC instability, we find that $\mathcal{A}_{\mathrm{e}}>1$ reduces the growth rate (see the red and black lines in panel (a) and also Ahmadi et al. 2016). By contrast, the whistler instability is unstable for $\mathcal{A}_{\mathrm{e}}>1$, and the growth rate is independent of $\mathcal{A}_{\mathrm{i}}$. The whistler mode is also quasi-parallel-propagating with $\theta_{m}=0^{\circ}$. The mirror modes, on the other hand, are highly oblique with $\theta_{m}=64^{\circ}$ for LM3.0 $\beta 50-\mathrm{m} 1836$. The e-mirror mode (blue) at high $k\left(c k / \omega_{\mathrm{pi}}>0.3\right)$ grows much faster than the i-mirror mode (red) at low $k\left(c k / \omega_{\mathrm{pi}}<0.3\right)$. With both $\mathcal{A}_{\mathrm{i}}>1$ and $\mathcal{A}_{\mathrm{e}}>1$, a mixture of the two mirror modes appears in the intermediate- $k$ regime $\left(c k / \omega_{\mathrm{pi}} \sim 0.3\right)$.

In the LM3.0 $\beta 50-\mathrm{m} 1836$ model, the maximum growth rates are given in the following order:

$$
\gamma_{\mathrm{WI}} \gg \gamma_{\mathrm{EM}} \gg \gamma_{\mathrm{AIC}}>\gamma_{\mathrm{IM}},
$$

where $\gamma_{\mathrm{WI}}, \gamma_{\mathrm{EM}}, \gamma_{\mathrm{AIC}}$, and $\gamma_{\mathrm{IM}}$ are the maximum growth rates of the whistler, e-mirror, AIC, and i-mirror instabilities, respectively. Note that, in general, $\gamma_{\mathrm{WI}}>\gamma_{\mathrm{EM}}$ (Gary \& Karimabadi 2006), and $\gamma_{\text {AIC }}>\gamma_{\text {IM }}$ under space plasma conditions with low $\beta$ and large temperature anisotropies (Gary 1992, 1993).

The real frequency, $\omega_{r} / \Omega_{\mathrm{ci}}$, at $\theta_{m}$ for the mixed case $\left(\mathcal{A}_{\mathrm{e}}=1.2\right.$ and $\mathcal{A}_{\mathrm{i}}=2.0$ ) is shown in panels (d)-(f) of Figure 2. The AICdriven mode has $\omega_{r} / \Omega_{\mathrm{ci}} \sim 0.25-0.5$ for $c k / \omega_{\mathrm{pi}} \sim 0.1-0.4$, while the whistler mode has $\omega_{r} / \Omega_{\mathrm{ci}} \sim 80-350$ for $c k / \omega_{\mathrm{pi}} \sim 5-20$. The mirror modes are nonpropagating or purely growing with $\omega_{r}=0$. Moreover, the polarization, calculated using the solutions of the dispersion relation, is $P=-1,+1$, and 0 for the AIC, whistler, and mirror instabilities, respectively, as expected.

\subsection{Parameter Dependence of Linear Properties}

As listed in Table 2, we consider a number of models to explore the dependence on $m_{\mathrm{i}} / m_{\mathrm{e}}$ and $\beta$. The upper panels of Figure 3 show the linear predictions for the models with 


\section{LM3.0 $350-\mathrm{m} 1836\left(\beta_{\mathrm{e}}=18.9, \beta_{\mathrm{i}}=31.1, \mathrm{~m}_{\mathrm{i}} / \mathrm{m}_{\mathrm{e}}=1836, \omega_{\mathrm{pe}} / \Omega_{\mathrm{ce}}=26\right)$}
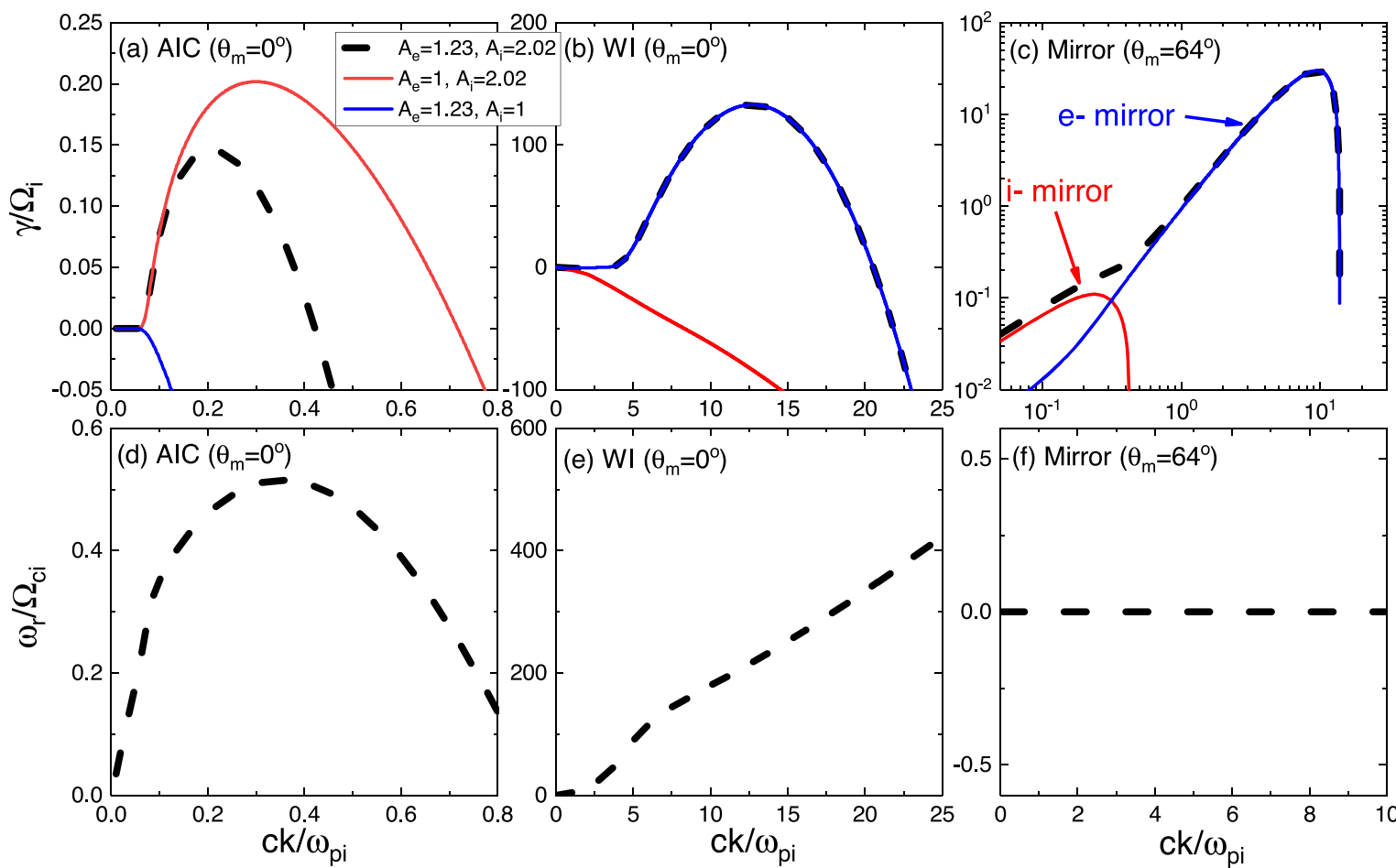

(e) WI $\left(\theta_{m}=0^{\circ}\right)$

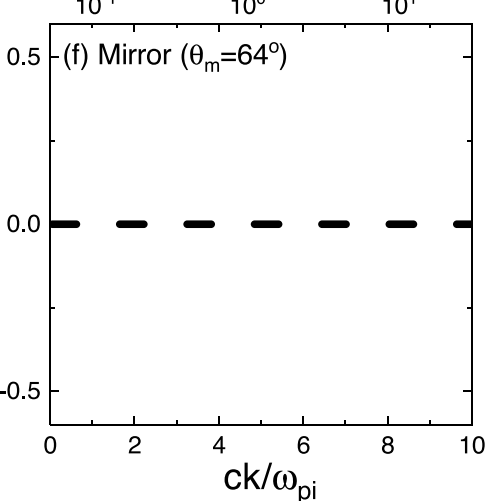

Figure 2. (a)-(c) Linear growth rate, $\gamma$, at the propagation angle of the fastest-growing mode, $\theta_{m}$, for the AIC, whistler, and mirror modes, respectively, as a function of the wavenumber $k$ for the LM3.0 350 -m1836 model. To examine separately the electron mode (blue) and the ion mode (red), the cases of $\mathcal{A}_{\mathrm{e}}=1.2$ and $\mathcal{A}_{\mathrm{i}}=1.0$ (blue) and $\mathcal{A}_{\mathrm{e}}=1.0$ and $\mathcal{A}_{\mathrm{i}}=2.0$ (red) are shown. The black dashed lines show the mixed-mode case, in which $\mathcal{A}_{\mathrm{e}}=1.2$ and $\mathcal{A}_{\mathrm{i}}=2.0$. In panel (c), both $\gamma$ and $k$ are plotted in logarithmic scales. (d)-(f) Real frequency, $\omega_{r}$, for the same case as the black dashed lines in the upper panels. Note that $\gamma$ and $\omega_{r}$ are normalized by $\Omega_{\mathrm{ci}}$, and $k$ is normalized by $\omega_{\mathrm{pi}} / c$, uniformly for both the ion and electron modes.
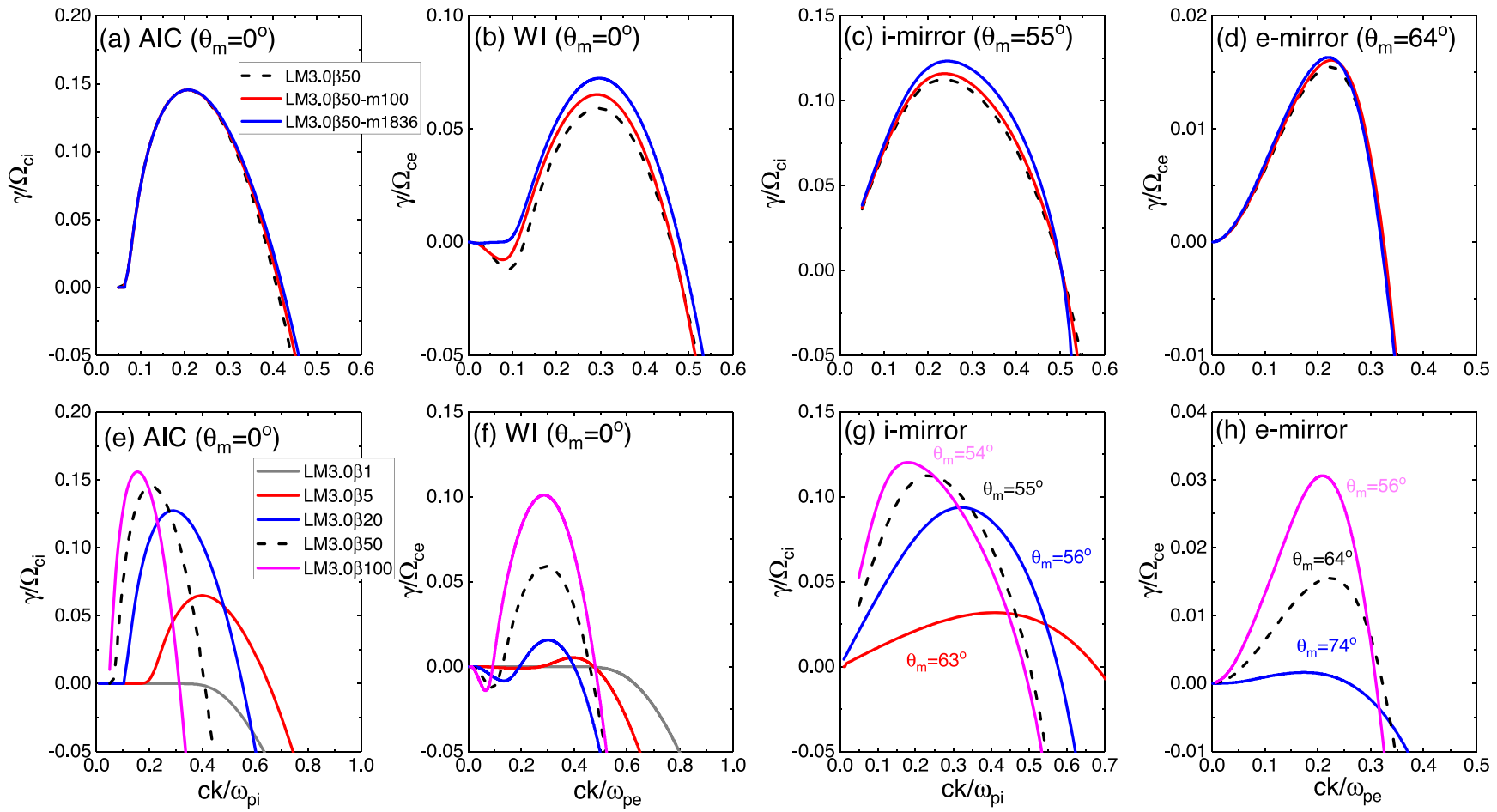

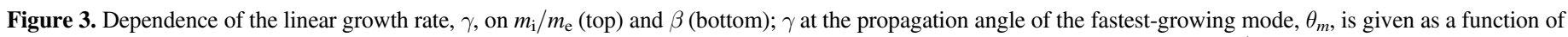
the wavenumber $k$. The model parameters are listed in Table 2 . Note that for the mirror modes, $\theta_{m}$ depends on $\beta$, but not on $m_{\mathrm{i}} / m_{\mathrm{e}}$. 

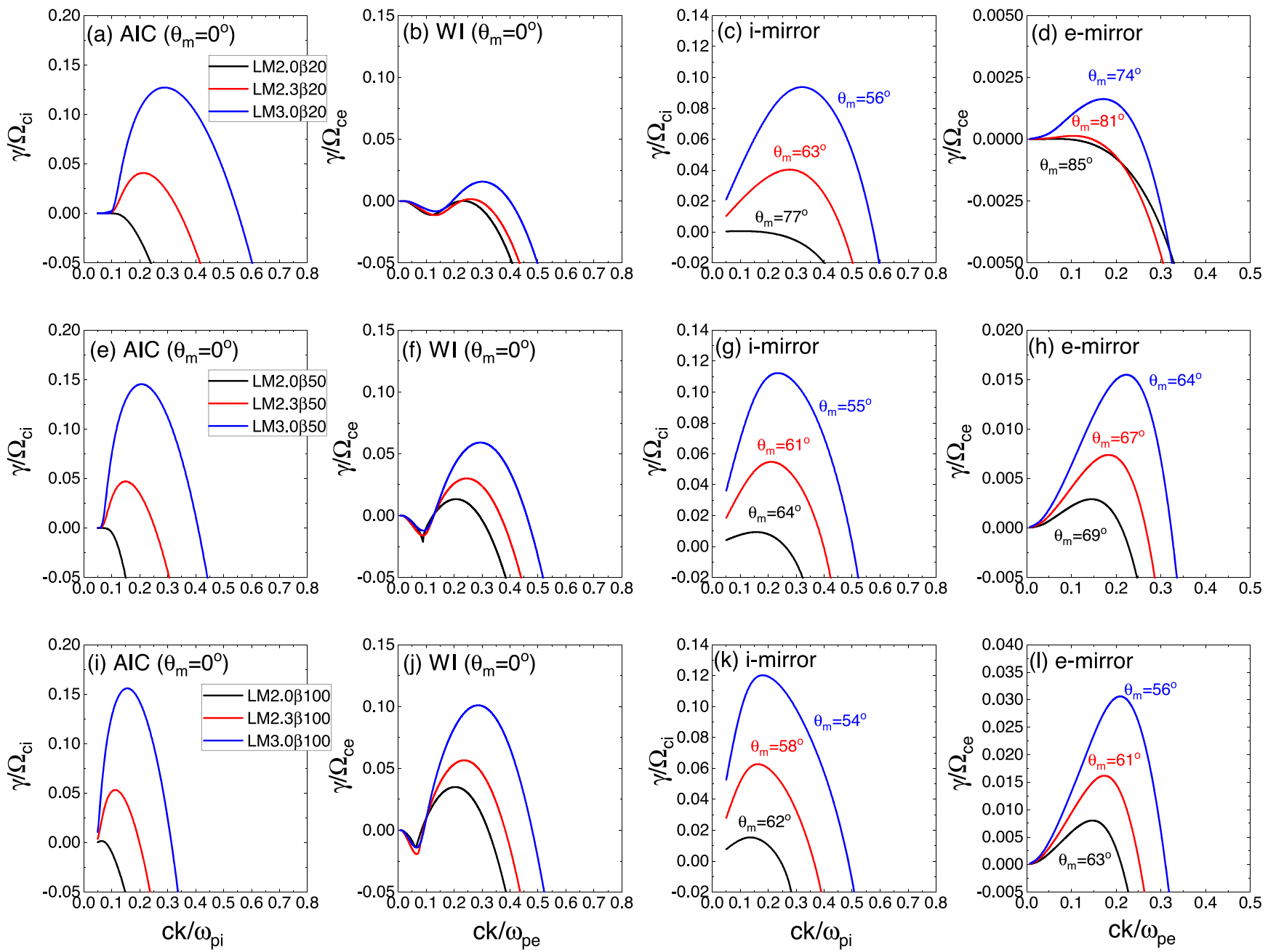

Figure 4. Dependence of the linear growth rate, $\gamma$, on $M_{\mathrm{s}}$ and $\beta ; \gamma$ at the propagation angle of the fastest-growing mode, $\theta_{m}$, is given as a function of the wavenumber $k$. In each panel, the black, red, and blue lines show the results for $M_{\mathrm{s}}=2.0,2.3$, and 3.0, respectively. The plasma beta varies as $\beta=20$ (top), 50 (middle), and 100 (bottom). The model parameters are listed in Table 2. Note that for the mirror modes, $\theta_{m}$ depends on $\beta$.

$M_{\mathrm{s}}=3, \beta=50$, and $m_{\mathrm{i}} / m_{\mathrm{e}}=50-1836$, while the lower panels are for the models with $M_{\mathrm{s}}=3, m_{i} / m_{e}=50$, and $\beta=1-100$. For a higher mass ratio, electrons go through more gyromotions per the ion gyrotime, $\Omega_{\mathrm{ci}}^{-1}$. Nevertheless, $\gamma_{\mathrm{AIC}} / \Omega_{\mathrm{ci}}$ and $\gamma_{\mathrm{EM}} / \Omega_{\mathrm{ce}}$ are almost independent of $m_{\mathrm{i}} / m_{\mathrm{e}}$. In the case of the whistler and i-mirror instabilities, on the other hand, overall, the normalized growth rates are slightly lower for smaller $m_{i} / m_{e}$. Also, the damping rate for the whistler instability is slightly higher for smaller $m_{i} / m_{e}$ in the small wavenumber regime $\left(c k / \omega_{\mathrm{pe}} \sim 0.1\right)$. As a result, the growth of the whistler and i-mirror instabilities may be somewhat suppressed in the shock simulations with reduced mass ratios. However, even in the case of $m_{\mathrm{i}} / m_{\mathrm{e}}=50$, this effect is expected to only be minor, because the inequality in Equation (4) is still valid and the changes of $k_{m}$ and $\theta_{m}$ are negligible (see Table 2).

The plasma beta is another important parameter that affects the stability of the system. Note that the anisotropy parameters, $\mathcal{A}_{\mathrm{e}}$ and $\mathcal{A}_{\mathrm{i}}$, are almost independent of $\beta$ for $\beta \approx 20-100$, the range relevant for ICM shocks (see Table 2), although they tend to increase slightly with increasing $\beta$ in the second digit to the right of the decimal point. In the low- $\beta$ case (LM3.0 $\beta 1), \mathcal{A}_{\mathrm{i}}=1.2$ is significantly smaller than that of other high- $\beta$ models due to the strong magnetization of ions. This is because $\mathcal{A}_{\mathrm{i}}$ in the shock transition is closely related to the fraction of reflected ions. On the other hand, $\mathcal{A}_{\mathrm{e}}$ in the shock transition is not substantially affected by $\beta$, because it is mainly determined by the magnetic field compression rather than the fraction of reflected electrons. Given the same temperature anisotropies, the growth of the instabilities tends to be suppressed by strong magnetic fields at low- $\beta$ plasmas. As can be seen in the lower panels of Figure 3, the peak values of either $\gamma_{m} / \Omega_{\mathrm{ci}}$ for the ion-driven modes or $\gamma_{m} / \Omega_{\mathrm{ce}}$ for the electrondriven modes increase with increasing $\beta$. For the AIC, whistler, and i-mirror instabilities, $\gamma_{m} / \Omega_{\mathrm{ci}}$ or $\gamma_{m} / \Omega_{\mathrm{ce}}$ occurs at smaller $c k / \omega_{\mathrm{pi}}$ or $c k / \omega_{\mathrm{pe}}$, for higher $\beta$. But such a trend is not obvious in the case of the e-mirror mode.

In the high- $\beta$ cases $(\beta \approx 20-100)$ with $M_{\mathrm{s}}=3$, all of the AIC, whistler, i-mirror, and e-mirror waves can be triggered, as shown in the lower panels of Figure 3, leading to the generation of multiscale waves from electron to ion scales. On the other hand, in the LM3.0/35 model (red solid lines), the e-mirror mode is stable, but other modes are unstable. In the LM3.0 31 model (gray solid lines), all instabilities are stable with negative growth rates.

The sonic Mach number, $M_{\mathrm{s}}$, is the key parameter that determines the temperature anisotropies in the transition of high- $\beta$ ICM shocks $(\beta \approx 20-100)$, because the ion reflection fraction and the magnetic field compression are closely related to $M_{\mathrm{s}}$. Figure 4 shows the growth rates of the instabilities for $M_{\mathrm{s}}=2.0$ (black), 2.3 (red), and 3.0 (blue) in the cases of 
$\beta=20$ (top), 50 (middle), and 100 (bottom). As $M_{\mathrm{s}}$ increases, both $\mathcal{A}_{\mathrm{e}}$ and $\mathcal{A}_{\mathrm{i}}$ increase, so all the modes grow faster and $k_{m}$ shifts toward larger $k$, regardless of $\beta$.

Note that the AIC and whistler modes have $\gamma_{m}$ at $\theta_{m}=0$ independent of $M_{\mathrm{s}}$, whereas $\theta_{m}$ decreases with increasing $M_{\mathrm{s}}$ for the i-mirror and e-mirror modes (see also Table 2). In LM2.0 $\beta 50$ and LM2.0 $\beta 100$, the AIC instability is stable or quasi-stable, while the whistler and mirror modes can grow. In the case of LM2.0 $\beta 20$, all of the instabilities are stable (see black lines in top panels). In the models with $M_{\mathrm{s}}=2.3-3$ (red and blue lines), on the other hand, the four instabilities are unstable, and hence multiscale plasma waves can be generated.

The parameter dependence can be summarized as follows. (1) For the AIC mode, the maximum normalized growth rate, $\gamma_{m} / \Omega_{\mathrm{ca}}$, and the corresponding normalized wavenumber, $c k_{m} / \omega_{\text {pa }}$, are almost independent of $m_{i} / m_{e}$. For the whistler, i-mirror, and e-mirror modes, on the other hand, $\gamma_{m} / \Omega_{\mathrm{ca}}$ is only slightly enhanced for larger $m_{i} / m_{e}$, whereas $c k_{m} / \omega_{\mathrm{pa}}$ exhibits almost no dependence. (2) For all the modes, the overall trend shows that $\gamma_{m} / \Omega_{\mathrm{ca}}$ is higher and $c k_{m} / \omega_{\mathrm{pa}}$ is smaller for higher $\beta$. (3) For all the modes, $\gamma_{m} / \Omega_{\mathrm{ca}}$ is higher and $c k_{m} / \omega_{\mathrm{pa}}$ is larger for higher- $M_{\mathrm{s}}$ cases with larger $\mathcal{A}_{\mathrm{e}}$ and larger $\mathcal{A}_{\mathrm{i}}$.

\section{Nonlinear Evolution of Induced Waves in Periodic-box Simulations}

\subsection{Numerical Setup}

To investigate the development and nonlinear evolution of the instabilities, we performed 2D PIC simulations with periodic boundary conditions for the three fiducial models, LM2.0 $\beta 50$, LM2.3 $\beta 50$, and LM3.0 $\beta 50$, with the same setup described in Section 2.1. Electrons and ions are prescribed with bi-Maxwellian VDFs with $\beta_{\mathrm{e}}, \beta_{\mathrm{i}}, \mathcal{A}_{\mathrm{e}}$, and $\mathcal{A}_{\mathrm{i}}$ given in Table 2. As noted before, here $m_{\mathrm{i}} / m_{\mathrm{e}}=50$ is employed due to computational limitations, but at least the early, linear-stage development of the plasma instabilities under consideration is expected to depend rather weakly on the mass ratio.

We point out that the setup for these 2D periodic-box simulations should intrinsically differ from the condition in the transition zone of shocks in the following aspects. (1) The initial distributions of ions and electrons are allowed to relax in the periodic-box simulations. By contrast, the shock-reflected ions and electrons are continuously convected into the transition zone behind the shock ramp, leading to the continuous excitation of the instabilities. (2) Homogeneous spatial distributions and bi-Maxwellian VDFs are assumed for the periodic-box simulations. On the other hand, as noted in Section 2.1 , both the ion and electron distributions are spatially nonuniform and the VDFs of ions and electrons contain suprathermal tails in the shock transition region. Nevertheless, periodic-box simulations like ours are often used to investigate the nonlinear evolution and properties of microinstabilities in either the upstream or downstream region near the shock (e.g., Scholer et al. 2000; Guo et al. 2014b; Trotta et al. 2020).

The simulations were carried out using a parallelized EM PIC code, TRISTAN-MP (Buneman 1993; Spitkovsky 2005). The simulation domain is a square of box size $L_{\mathrm{x}}=L_{\mathrm{z}}=84.8 \mathrm{c} /$ $\omega_{\mathrm{pi}}=600 \mathrm{c} / \omega_{\mathrm{pe}}$ in the $x-z$ plane, which consists of grid cells of $\Delta x=\Delta z=0.1 c / \omega_{\text {pe }}$. In each cell, 32 particles (16 for ions and 16 for electrons) are placed. The time step of the simulations is $\Delta t=0.045 \omega_{\mathrm{pe}}^{-1}$, and the simulations ran up to $t_{\mathrm{end}}=130 \Omega_{\mathrm{ci}}^{-1}$. Interpreting the results of our PIC simulations could be limited by numerical noises and aliases due to a finite number of macroparticles on discretized grids (e.g., Pohl et al. 2020). However, we expect that the overall results of the PIC simulations are reasonably converged, judging from the previous studies (Guo et al. 2014b; Kang et al. 2019; Kim et al. 2020).

\subsection{Results of Periodic-box Simulations}

With the inequality in Equation (4), we expect that the whistler mode grows much faster than other modes, resulting in the relaxation of $\mathcal{A}_{\mathrm{e}}$ during the early stage. As the whistler and e-mirror modes grow and then decay on the timescale of $\tau_{\mathrm{WI}} \equiv 1 / \gamma_{\mathrm{WI}}$, the AIC and i-mirror modes become dominant later on the timescale of $\tau_{\text {AIC }} \equiv 1 / \gamma_{\text {AIC }}$.

Figure 5 shows the magnetic field fluctuations, $\delta B_{y}$ (upper panels) and $\delta B_{z}$ (lower panels), in the $x-z$ plane (simulation plane) at three different times in the LM3.0 $\beta 50$ model. Here, the growth timescales, $\tau_{\mathrm{WI}}$ and $\tau_{\mathrm{AIC}}$, are estimated with the $\gamma_{m}$ of each mode in Table 2 . At $t \sim \tau_{\mathrm{WI}}$, the transverse component, $\delta B_{y}$, appears on electron scales, and the waves containing it propagate parallel to $\boldsymbol{B}_{0}$ in panel (a), but the longitudinal component, $\delta B_{z}$, does not grow significantly in panel (d). In this early stage, the dominant mode is the whistler mode, while the e-mirror mode is too weak to be clearly identified. As $\mathcal{A}_{\mathrm{e}}$ decreases in time due to the electron scattering off the excited waves, the whistler waves decay as shown in panel (b). On the timescale of $\tau_{\text {AIC }}$, both the AIC and i-mirror instabilities grow and become dominant. It is clear that the AIC-driven waves, shown in panel (c), are parallel-propagating, while the i-mirrordriven waves, shown in panel (f), are oblique-propagating; the blue arrow in the bottom-left corner of panel (f) denotes the wavevector of the i-mirror-driven mode with the maximum growth rate.

Figure 6 shows the time evolution of the power spectrum for the magnetic field fluctuations, $\delta B_{y}^{2}(\boldsymbol{k})$, for LM2.0 $\beta 50$, $\mathrm{LM} 2.3 \beta 50$, and LM3.0 $\beta 50$ at $t \sim \tau_{\mathrm{WI}}, t \sim 3 \tau_{\mathrm{WI}}$, and $t \sim \tau_{\mathrm{AIC}}$. Again, the growth timescale of each mode is estimated with $\gamma_{m}$ listed in Table 2, except for the LM2.0 350 model, in which the AIC instability is stable and so the output time of panel (i) is chosen at the evolutionary stage similar to that of LM2.3 $\beta 50$. In the cases of $M_{\mathrm{s}}=2.3$ and 3 , whistler waves are excited dominantly at quasi-parallel-propagating angles at $t \sim \tau_{\mathrm{WI}}$. After the initial linear stage, the energy of the whistler waves is transferred to smaller wavenumbers and the waves gradually decay, as shown in panels (b) and (e). On the timescale of $\sim \tau_{\text {AIC }}$, AIC waves and i-mirror waves appear dominantly at quasi-parallel and highly oblique angles, respectively, as shown in panels (c) and (f). This is consistent with the evolutionary behavior that we have described in Figure 5. For the AIC and whistler instabilities, the linear predictions for $\boldsymbol{k}_{m}$ with the maximum growth rate (gray star symbols) agree reasonably well with the peak locations of the magnetic power spectrum realized in the PIC simulations. But the linear estimates for the i-mirror mode are slightly off because $\gamma_{m}$ is obtained without electron anisotropy, as stated in Section 2.2. In summary, the results of the periodic-box simulations are quite consistent with the linear predictions described earlier. Also, we note that the results of our PIC simulations are in good agreement with those of Ahmadi et al. (2016), in which PIC simulations were carried out to explore the evolution of the instabilities due to the temperature anisotropies in space plasmas with $\beta \sim 1$. The bottom panels of Figure 6 confirm that waves do not grow noticeably in the LM2.0 $\beta 50$ model. 

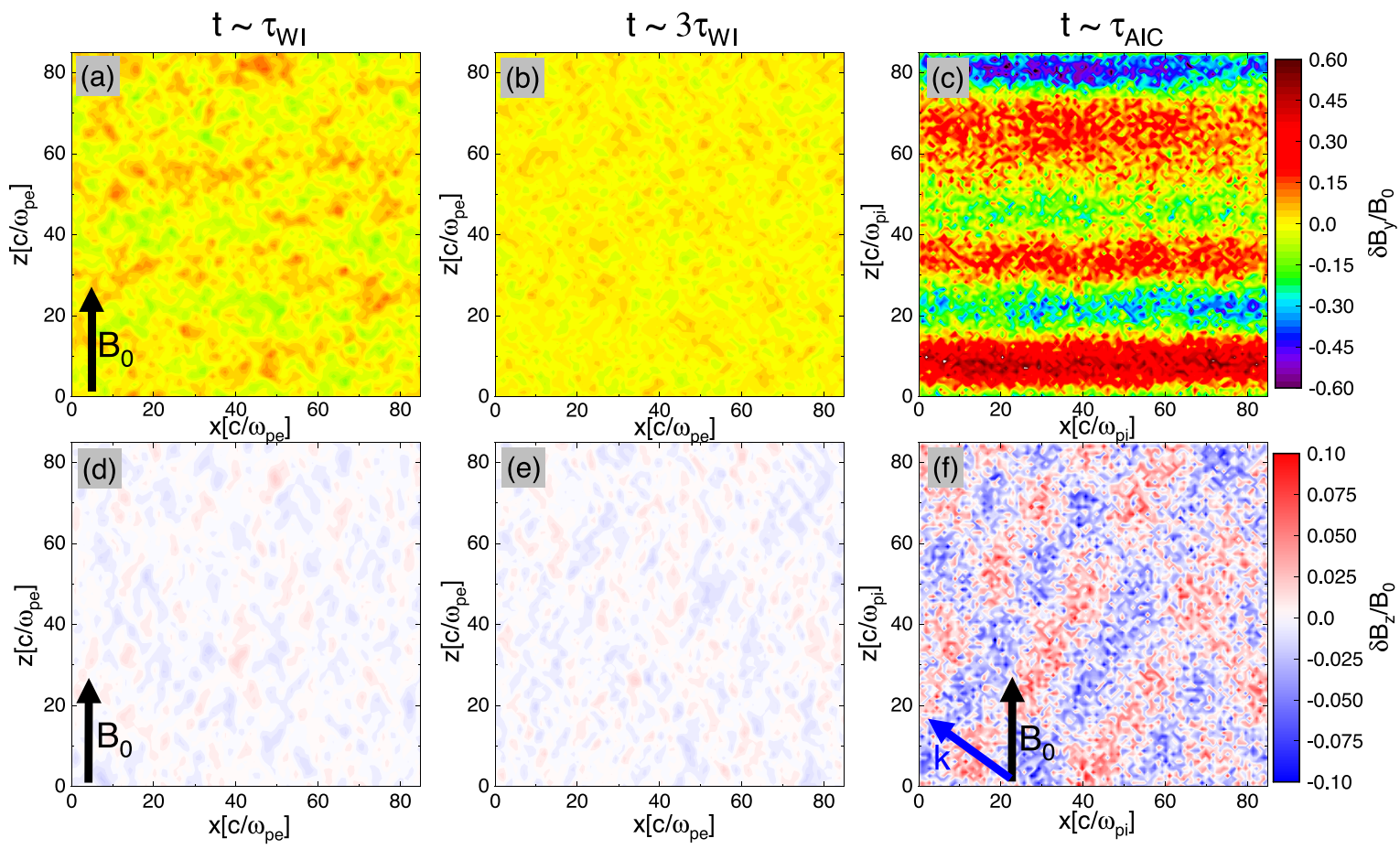

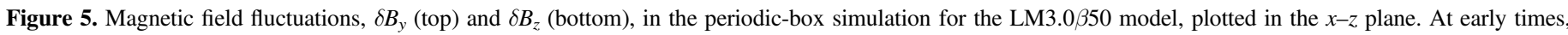

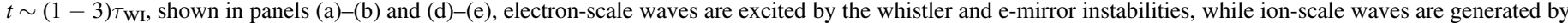

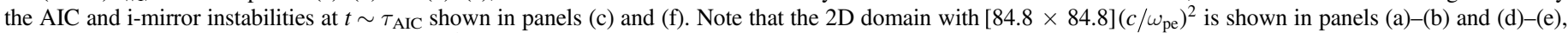

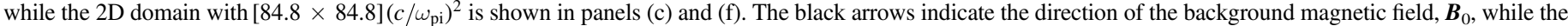
blue arrow in panel (f) shows the direction of wave propagation, $\boldsymbol{k}$, for the i-mirror mode with the maximum growth rate.

In these periodic-box simulations, the electron-scale waves develop first and then decay as $\mathcal{A}_{\mathrm{e}}$ is relaxed in the early stage, followed by the growth of the ion-scale waves due to $\mathcal{A}_{\mathrm{i}}$. In the shock transition region, by contrast, temperature anisotropies are to be supplied continuously by newly reflected-gyrating ions and magnetic field compression, hence multiscale plasma waves from electron to ion scales are expected to be simultaneously present.

\section{Implications for Shock Simulations}

\subsection{Shock Criticality}

As mentioned in the introduction, the Fermi-like acceleration, which relies on the upstream waves excited by the EFI, is effective only for supercritical shocks with $M_{\mathrm{s}} \geqslant M_{\mathrm{EFI}}^{*} \approx 2.3$ in $\beta \approx 20-100$ plasma (Guo et al. 2014b; Kang et al. 2019). The SSDA, which depends on the multiscale waves excited mainly by the AIC and whistler instabilities, is thought to occur in supercritical shocks with $M_{\mathrm{s}} \geqslant M_{\mathrm{AIC}}^{*} \approx 3.5$ in $\beta \approx 1$ plasmas (Trotta \& Burgess 2019) and $M_{\mathrm{s}} \geqslant M_{\mathrm{AIC}}^{*} \approx 2.3$ in $\beta \approx 20-100$ plasmas (HKRK2021). We suggest that both $M_{\mathrm{EFI}}^{*}$ and $M_{\mathrm{AIC}}^{*}$ are related to the sonic critical Mach number, $M_{\mathrm{s}}^{*}$, for ion reflection, because the structure of collisionless shocks is governed primarily by the dynamics of shock-reflected ions.

Table 3 summarizes the shock criticality of the simulated shock models and the stability of the linear analysis models. The first column lists the name of the simulated shock models considered in HKRK2021 and the two additional models for low- $\beta$ shocks performed for this study. The shock criticality of each model is given in the second column. The name of the corresponding linear analysis models is given in the third column, while the last four columns show the stability for the four instabilities (see also Table 2). We note that the name of the shock models includes $\beta^{\text {up }}$ in the preshock, upstream plasmas, while that of the linear analysis models includes $\beta$ in the shock transition zone given in Table 2 .

As discussed in Sections 2 and 3, in $\beta \approx 20-100$ plasmas, the AIC instability operates for $M_{\mathrm{s}} \gtrsim 2.3$, while whistler waves are induced regardless of $M_{\mathrm{s}}$. In the M3.0 35 model, the e-mirror mode is stable, while the other three modes are unstable. This is in good agreement with the 2D simulation of an $M_{s}=5$ and $\beta=5$ shock reported earlier by Niemiec et al. (2019). In the M3.0 $\beta 1$ model, by contrast, $\mathcal{A}_{\mathrm{i}}$ is smaller than that of high- $\beta$ models, and all four instability modes are suppressed by strong magnetization. This is consistent with the results of $M_{\mathrm{AIC}}^{*} \approx 3.5$ for shocks with $\beta \approx 1$ presented by Trotta \& Burgess (2019).

\subsection{Shock Surface Rippling}

Another important feature of supercritical shocks above $M_{\mathrm{AIC}}^{*}$ is the shock surface rippling. According to previous shock simulations (e.g., Lowe \& Burgess 2003; Matsukiyo \& Matsumoto 2015; Niemiec et al. 2019; Trotta \& Burgess 2019), the rippling has the characteristics of AIC waves with the fastest-growing mode at $\theta_{m} \sim 0$, the propagation speed close to the local Alfvén speed, and the wavelengths of $\sim \lambda_{\mathrm{AIC}}$ $\left(\approx 30 c / \omega_{\mathrm{pi}}\right)$. In fact, shock ripples have been observed at Earth's bow shock and interplanetary shocks inside the heliosphere and investigated extensively in space physics (e.g., Winske \& Quest 1988; Moullard et al. 2006; Johlander et al. 2016). At the interplanetary shocks, ripples on scales even larger than $\lambda_{\text {AIC }}$ have been detected as well and are thought to be triggered by the upstream magnetic structures produced by backstreaming ions (Kajdič et al. 2019). 

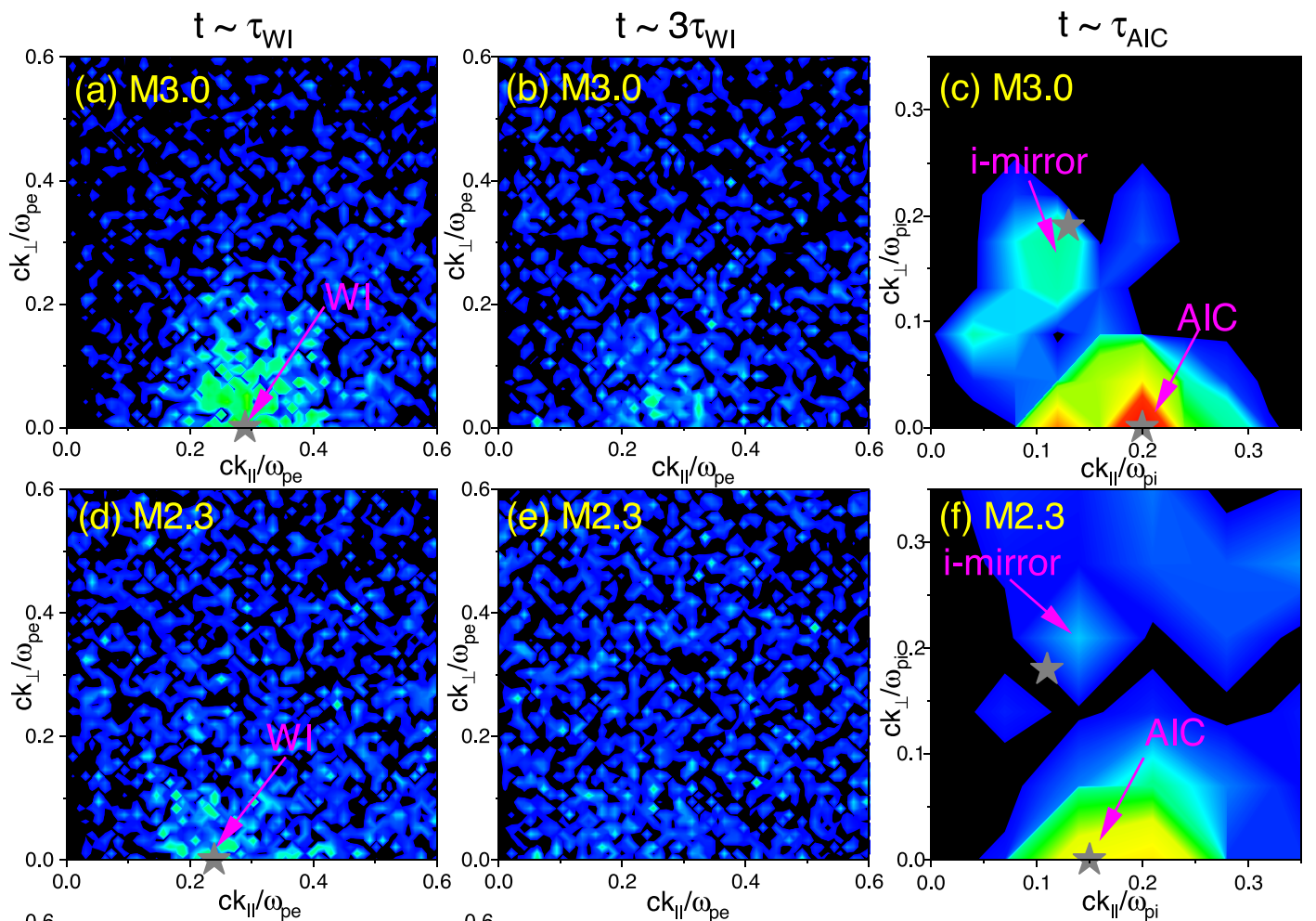

$10^{-3}$
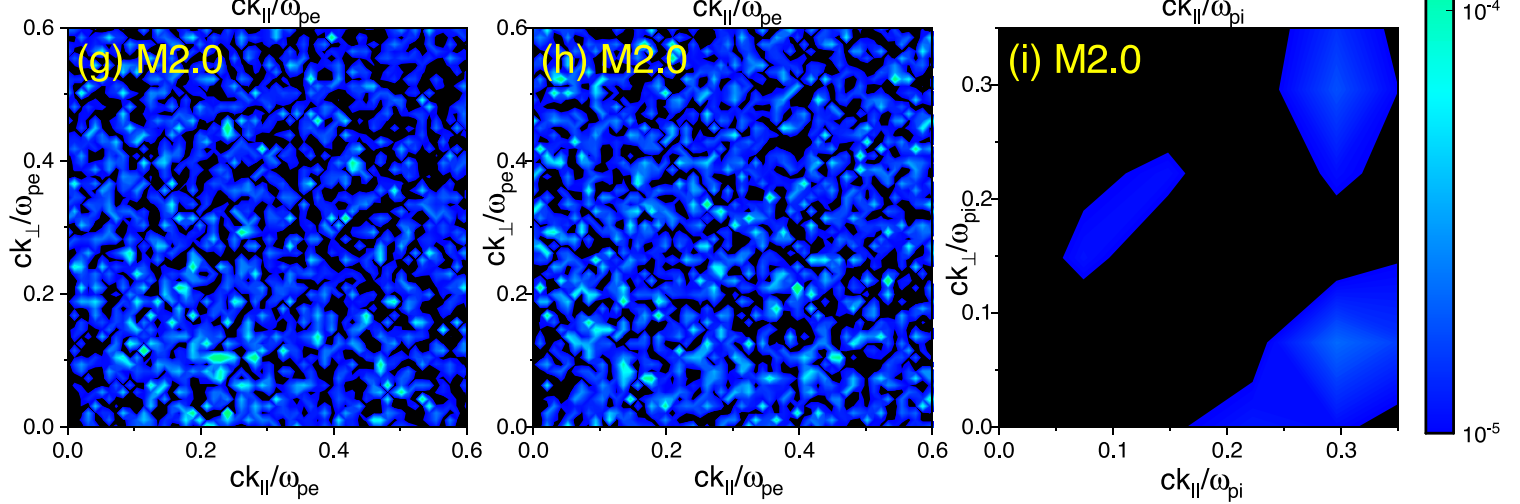

Figure 6. Power spectra of the magnetic field fluctuations, $\delta B_{y}^{2}(\boldsymbol{k})$, in the periodic-box simulations for LM3.0 350 (top), LM2.3350 (middle), and LM2.0 350 (bottom), plotted in the $k_{\|}-k_{\perp}$ (that is, $k_{z}-k_{x}$ ) plane. The results are shown at $t \sim \tau_{\mathrm{WI}}$ (left), $t \sim 3 \tau_{\mathrm{WI}}$ (middle), and $t \sim \tau_{\mathrm{AIC}}$ (right). See the text for the remarks on $\tau_{\mathrm{AIC}}$ for LM2.0 350 . The gray star symbol marks the location of the maximum linear growth rate, $\gamma_{m}$, estimated from the linear analysis. In the models with $M_{\mathrm{s}} \geqslant 2.3$, AIC, whistler, and i-mirror waves appear, while those waves do not grow substantially in the model with $M_{\mathrm{s}}=2$.

The parallel-propagating AIC and whistler waves in homogeneous plasmas are purely electromagnetic and incompressible, with both the electric and magnetic wavevectors pointing normal to $\boldsymbol{B}_{0}$. The fluctuating magnetic fields of oblique mirror modes, on the other hand, have a substantial longitudinal component, that is, $\delta \boldsymbol{B}$ has a significant component parallel to $\boldsymbol{B}_{0}$ (Gary 1993). Because the density fluctuations are proportional to the parallel electric and magnetic field fluctuations (Hojo et al. 1993), we expect to see only weak ion-density fluctuations due to the i-mirror mode in our 2D periodic-box simulations.

Panel (b) of Figure 7 displays the variations of the ion density, $\left[\left\langle n_{\mathrm{i}}-n_{0}\right\rangle_{\mathrm{x}, \mathrm{avg}} / n_{0}\right] \approx \pm 0.01$, averaged over the $x$ direction in the periodic-box simulation for the LM3.0 350 model. Panel (a) shows the fluctuations of the transverse component of $\boldsymbol{B}_{0},\left[\left\langle B_{y}-B_{0}\right\rangle_{\mathrm{x}, \text { avg }} / B_{0}\right] \approx \pm 0.4$, which have a relatively large amplitude due to the AIC-driven waves. It shows that even after the AIC-driven waves have fully grown, they have little effect on the ion-density fluctuations.
However, the ion-density fluctuations of the rippling waves propagating along the shock surface behind the shock ramp are rather significant in the shock simulation for the M3.08350 model in HKRK2021. Panel (c) shows that both the variations of $\left[\left\langle n_{\mathrm{i}}-n_{0}\right\rangle_{\mathrm{x}, \mathrm{avg}} / n_{0}\right] \approx \pm 0.1$ and $\left[\left\langle B_{y}-B_{0}\right\rangle_{\mathrm{x}, \mathrm{avg}} / B_{0}\right] \approx \pm 0.1$ have similar amplitudes; the fluctuations of $n_{\mathrm{i}}$ are much larger than those of the linear prediction expected for the parallelpropagating AIC mode. Note that here the quantities are averaged along the $x$ direction over the shock transition zone including the first and second overshoot oscillations behind the ramp. Hence, the basic assumptions of the linear theory, such as the homogeneous background, charge neutrality, and zero net current, are likely to be violated in this region.

We point out that such large-amplitude fluctuations of $n_{\mathrm{i}}$, comparable to the fluctuations of $B_{y}$, were previously recognized in the 2D hybrid simulations of supercritical, perpendicular shocks presented in Winske \& Quest (1988). The authors suggested that the large compressive waves might result from nonlinear effects in addition to oblique i-mirror 
Table 3

Shock Criticality of the Simulated Shock Models and Stability of the Linear Analysis Models

\begin{tabular}{|c|c|c|c|c|c|c|}
\hline Simulated Shock Model & Shock Criticality & Linear Analysis Model & AIC & WI & Ion-mirror & Electron-mirror \\
\hline $\mathrm{M} 2.0 \beta 20$ & sub & LM2.0 $\beta 20$ & stable & stable & stable & stable \\
\hline $\mathrm{M} 2.0 \beta 50$ & sub & LM2.0 $\beta 50$ & stable & unstable & quasi-stable & unstable \\
\hline M2.0 $\beta 100$ & sub & LM2.0 $\beta 100$ & quasi-stable & unstable & unstable & unstable \\
\hline $\mathrm{M} 2.3 \beta 20$ & super & LM2.3 $\beta 20$ & unstable & unstable & unstable & stable \\
\hline $\mathrm{M} 2.3 \beta 50$ & super & LM2.3 $\beta 50$ & unstable & unstable & unstable & unstable \\
\hline M2.3 $\beta 100$ & super & LM2.3 $\beta 100$ & unstable & unstable & unstable & unstable \\
\hline M3.0 $\beta 1$ & sub & LM3.0 $\beta 1$ & stable & stable & stable & stable \\
\hline M3.0 $\beta 5$ & super & LM3.0 $\beta 5$ & unstable & unstable & unstable & stable \\
\hline M3.0 $\beta 20$ & super & LM3.0 $\beta 20$ & unstable & unstable & unstable & unstable \\
\hline $\mathrm{M} 3.0 \beta 50$ & super & LM3.0 $\beta 50$ & unstable & unstable & unstable & unstable \\
\hline M3.0 $\beta 100$ & super & LM3.0 $\beta 100$ & unstable & unstable & unstable & unstable \\
\hline
\end{tabular}

modes. The effects due to nonlinear couplings between various wave modes could be significant as well (e.g., Shukla \& Stenflo 1985; Verscharen \& Marsch 2011; Marsch \& Verscharen 2011). Therefore, the pure AIC-driven waves in the shock transition could have been modified by such possible nonlinearities, leading to the enhancement of ion-density fluctuations.

\section{Summary}

In supercritical $Q_{\perp}$ shocks, a substantial fraction of incoming ions and electrons are reflected, and the transverse components of magnetic fields are amplified at the shock ramp. The reflected-gyrating ions and the amplified magnetic fields induce the ion and electron perpendicular temperature anisotropies, $\mathcal{A}_{\mathrm{i}}$ and $\mathcal{A}_{\mathrm{e}}$, respectively, in the shock transition region (Guo et al. 2017). They in turn trigger various microinstabilities including the AIC, whistler, i-mirror, and e-mirror instabilities (e.g., Winske \& Quest 1988; Lowe \& Burgess 2003; Guo et al. 2017). The kinetic properties of these four instabilities are summarized in Table 1. The multiscale plasma waves generated by these microinstabilities are thought to be crucial for electron preacceleration via the SSDA (e.g., Katou \& Amano 2019; Niemiec et al. 2019; Trotta \& Burgess 2019).

In this work, adopting the numerical estimates for the ion and electron temperature anisotropies found in the 2D PIC simulations of $Q_{\perp}$ shocks with $M_{\mathrm{s}}=2-3$ (see Table 2), we have carried out the kinetic linear analysis of the microinstabilities for wide ranges of parameters, $\beta=1-100$ and $m_{i} / m_{e}=50-1836$. The linear predictions for the fastest-growing mode, $\gamma_{m}, k_{m}, \theta_{m}$, of each instability are given in Table 2 . In addition, in order to investigate the development and nonlinear evolution of the waves induced by the microinstabilities, we have performed 2D PIC simulations with periodic boundary conditions for the three fiducial models, LM2.0 $\beta 50$, LM2.3 $\beta 50$, and LM3.0 $\beta 50$. Finally, the results were also compared with the 2D PIC simulations for ICM shocks reported in HKRK2021.

The main results can be summarized as follows:

1. In the LM3.0 $\beta 50-\mathrm{m} 1836$ model with the real mass ratio, which represents a typical supercritical ICM shock, the maximum growth rates of the four instabilities have the following order: $\gamma_{\mathrm{WI}} \gg \gamma_{\mathrm{EM}} \gg \gamma_{\mathrm{AIC}}>\gamma_{\mathrm{IM}}$ (Figure 2). Hence, the parallel-propagating AIC and whistler waves are expected to be more dominant than the obliquepropagating mirror waves.
2. In the LM2.0 350 model, which represents a subcritical ICM shock, by contrast, the AIC mode is stable (Table 2), so mainly electron-scale whistler waves are generated.

3. The maximum normalized growth rates for the AIC and e-mirror instabilities, $\gamma_{\mathrm{AIC}} / \Omega_{\mathrm{ci}}$ and $\gamma_{\mathrm{EM}} / \Omega_{\mathrm{ce}}$, are almost independent of $m_{\mathrm{i}} / m_{\mathrm{e}}$, while $\gamma_{\mathrm{WI}} / \Omega_{\mathrm{ce}}$ for the whistler instability and $\gamma_{\mathrm{IM}} / \Omega_{\mathrm{ci}}$ for the i-mirror instability are slightly lower for smaller $m_{i} / m_{e}$ (Figure 3).

4. For all four instabilities, the maximum normalized growth rates increase with increasing $\beta$ (Figure 3).

5. As the sonic Mach number $M_{\mathrm{s}}$ increases, both $\mathcal{A}_{\mathrm{e}}$ and $\mathcal{A}_{\mathrm{i}}$ increase, all modes grow faster, and the $k_{m}$ of each mode shifts toward larger $k$, regardless of $\beta$ in the range of $\beta \approx 20-100$ (Figure 4).

6. The critical sonic Mach number to trigger the AIC instability in the shock transition is $M_{\mathrm{AIC}}^{*} \sim 2.3$ for $\beta \approx 20-100$. It is similar to the EFI critical number suggested in our previous study, that is, $M_{\mathrm{AIC}}^{*} \approx M_{\mathrm{ef}}^{*} \approx 2.3$ (Kang et al. 2019). For $\beta=1$, on the other hand, $M_{\mathrm{AIC}}^{*} \gtrsim 3$ is slightly higher, because the AIC mode is suppressed by the strong magnetization of ions (Hellinger \& Mangeney 1997; Trotta \& Burgess 2019).

7. The 2D periodic-box simulations confirm the linear predictions. In the early stage of $\sim \tau_{\mathrm{WI}}$, electron-scale waves develop and then decay as $\mathcal{A}_{\mathrm{e}}$ is relaxed, followed by the growth of ion-scale waves on the timescale of $\sim \tau_{\text {AIC }}$ (Figures 5 and 6).

8. The rippling waves propagating along the shock surface have the characteristics of AIC waves. Although the AIC waves are parallel-propagating, electromagnetic, and incompressible in the linear regime, the amplitudes of the longitudinal magnetic field and ion-density fluctuations associated with the overshoots in the shock transition are similar and of the order of $10 \%$ according to the shock simulation for the M3.0 350 model (Figure 7). It is expected that the inhomogeneity in the shock transition and the nonlinear effects could lead to the generation of such large-amplitude fluctuations of the ion density along the shock surface.

In conclusion, our results support well the suggestion for the generation of multiscale plasma waves via various microinstabilities in the transition region of high- $\beta$, supercritical, $Q_{\perp}$ shocks (Guo et al. 2017; Katou \& Amano 2019; Niemiec et al. 2019; Trotta $\&$ Burgess 2019). A detailed description of the shock structure and the electron preacceleration in such 


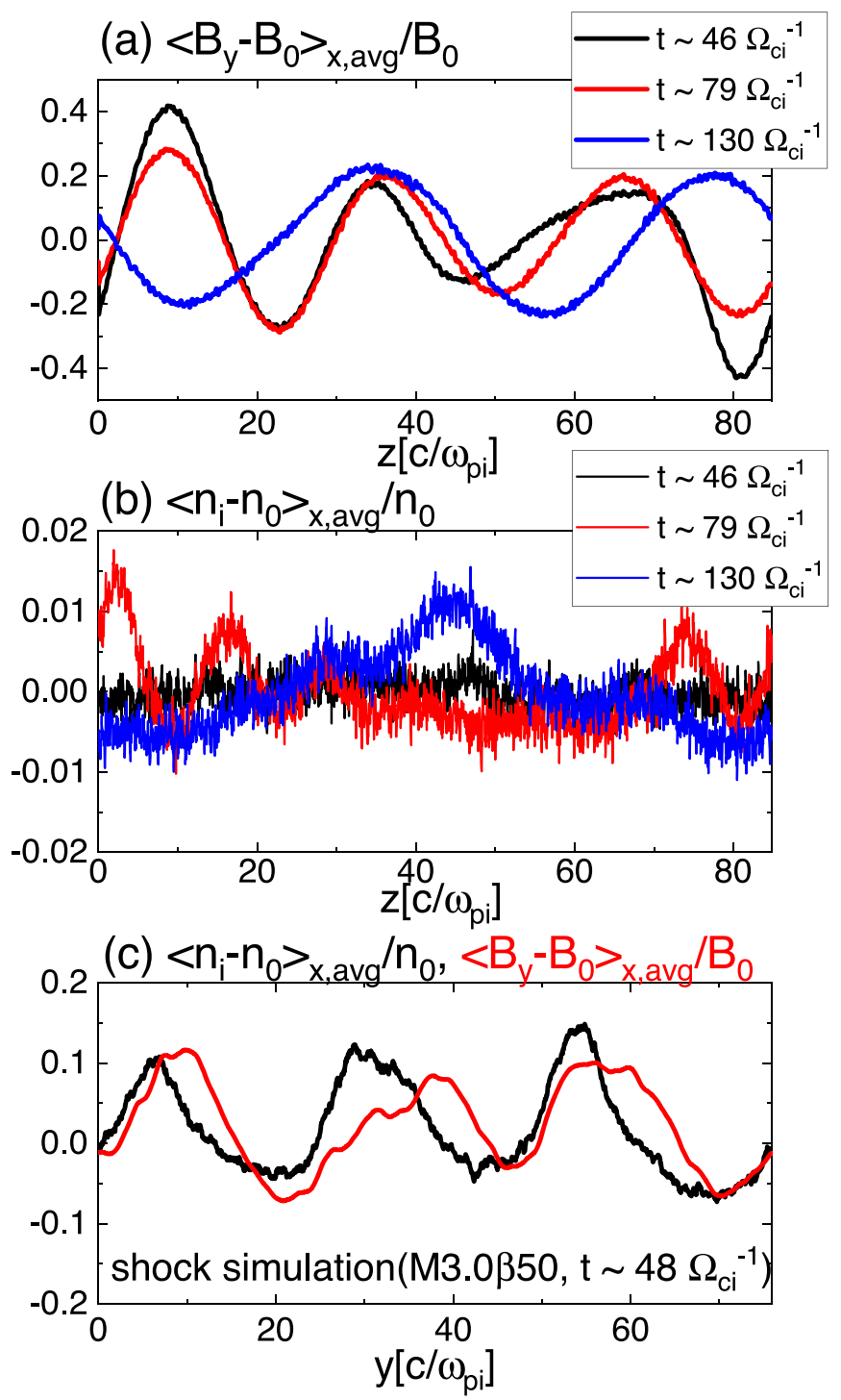

Figure 7. (a)-(b) Variations in the transverse component of the magnetic field, $\left\langle B_{y}-B_{0}\right\rangle_{\mathrm{x}, \text { avg }} / B_{0}$, and the ion density, $\left\langle n_{\mathrm{i}}-n_{0}\right\rangle_{\mathrm{x}, \text { avg }} / n_{0}$, averaged over the $x$ domain in the 2D periodic-box simulation for the LM3.0/350 model, plotted along $\boldsymbol{B}_{0}$ ( $z$ direction) at three different times. (c) Variations in the longitudinal component of the magnetic field, $\left\langle B_{y}-B_{0}\right\rangle_{\mathrm{x}, \mathrm{avg}} / B_{0}$ (red), and the ion density, $\left\langle n_{\mathrm{i}}-n_{0}\right\rangle_{\mathrm{x}, \mathrm{avg}} / n_{0}$ (black), averaged along the $x$ direction over the shock transition zone in the 2D shock simulation for the M3.0 $\beta 50$ model in HKRK2021, plotted along the $y$ direction. Note that the preshock magnetic field, $\boldsymbol{B}_{0}^{\text {up }}$, lies in the $x-y$ plane, and the obliquity angle between $\boldsymbol{B}_{0}^{\text {up }}$ and the $y$ axis is $\theta_{\mathrm{Bn}}=63^{\circ}$ in the shock simulation.

ICM shocks, realized in 2D PIC simulations, is reported in HKRK2021.

The authors thank the anonymous referee for constructive comments. This research used the high-performance computing resources of the UNIST Supercomputing Center. S.K. was supported by the NRF grant funded by the Korean government (MSIT)(NRF-2020R1C1C1012112). J.-H.H. and D.R. were supported by the National Research Foundation (NRF) of Korea through grants 2016R1A5A1013277 and 2020R1A2C2102800. J.-H.H. was also supported by the Global PhD Fellowship of the NRF through grant 2017H1A2A1042370. H.K. was supported by the Basic Science Research Program of the NRF through grant 2020R1F1A1048189.

\section{ORCID iDs}

Sunjung Kim (iD https://orcid.org/0000-0002-5441-8985

Ji-Hoon Ha (iD https://orcid.org/0000-0001-7670-4897

Dongsu Ryu (iD https://orcid.org/0000-0002-5455-2957

Hyesung Kang (iD https://orcid.org/0000-0002-4674-5687

\section{References}

Ahmadi, N., Germaschewski, K., \& Raeder, J. 2016, JGRA, 121, 5350

Brüggen, M., Bykov, A., Ryu, D., \& Röttgering, H. 2012, SSRv, 166, 187 Brunetti, G., \& Jones, T. W. 2014, IJMPD, 23, 1430007

Buneman, O. 1993, in Computer Space Plasma Physics, Simulation Techniques and Software, ed. H. Matsumoto \& Y. Omura (Tokyo: Terra Scientific), 67

Burgess, D. 2006, ApJ, 653, 316

Gary, S. P. 1992, JGRA, 97, 8519

Gary, S. P. 1993, Theory of Space Plasma Microinstabilities (Cambridge: Cambridge Univ. Press)

Gary, S. P., \& Karimabadi, H. 2006, JGRA, 111, A11224

Gary, S. P., \& Nishimura, K. 2003, PhPl, 10, 3571

Gary, S. P., Wang, J., Winske, D., \& Fuselier, S. A. 1997, JGR, 102, 27159 Gosling, J. T., Thomsen, M. F., Bame, S. J., \& Russell, C. T. 1989, JGR, 94, 10011

Guo, F., \& Giacalone, J. 2015, ApJ, 802, 97

Guo, X., Sironi, L., \& Narayan, R. 2014a, ApJ, 794, 153

Guo, X., Sironi, L., \& Narayan, R. 2014b, ApJ, 797, 47

Guo, X., Sironi, L., \& Narayan, R. 2017, ApJ, 851, 134

Ha, J.-H., Kim, S., Ryu, D., \& Kang, H. 2021, arXiv:2102.03042

Ha, J.-H., Ryu, D., \& Kang, H. 2018, ApJ, 857, 26

Hellinger, P., \& Mangeney, A. 1997, JGRA, 102, 9809

Hellinger, P., \& Štverák, Š 2018, JPIPh, 84, 905840402

Hojo, H., Mase, A., Katsumata, R., et al. 1993, JaJAP, 32, 3287

Hong, S. E., Ryu, D., Kang, H., \& Cen, R. 2014, ApJ, 785, 133

Johlander, A., Schwartz, S. J., Vaivads, A., et al. 2016, PhRvL, 117, 165101

Kajdič, P., Preisser, L., Blanco-Cano, X., Burgess, D., \& Trotta, D. 2019, ApJL, 874, L13

Kang, H., Ryu, D., \& Ha, J.-H. 2019, ApJ, 876, 79

Kang, H., Ryu, D., \& Jones, T. W. 2012, ApJ, 756, 97

Katou, T., \& Amano, T. 2019, ApJ, 874, 119

Kim, S., Ha, J.-H., Ryu, D., \& Kang, H. 2020, ApJ, 892, 85

Lowe, R. E., \& Burgess, D. 2003, AnGeo, 21, 671

Marsch, E., \& Verscharen, D. 2011, JPIPh, 77, 385

Matsukiyo, S., \& Matsumoto, Y. 2015, JPhCS, 642, 012017

Matsukiyo, S., Ohira, Y., Yamazaki, R., \& Umeda, T. 2011, ApJ, 742, 47

Moullard, O., Burgess, D., Horbury, T. S., \& Lucek, E. A. 2006, JGRA, 111, A09113

Niemiec, J., Kobzar, O., Amano, T., et al. 2019, in 36th Int. Cosmic Ray Conf. (ICRC2019) (Madison, WY), 36, 368

Oka, M., Terasawa, T., Seki, Y., et al. 2006, GeoRL, 33, L24104

Pohl, M., Hoshino, M., \& Niemiec, J. 2020, PrPNP, 111, 103751

Ryu, D., Kang, H., Hallman, E., \& Jones, T. W. 2003, ApJ, 593, 599

Scharer, J. E., \& Trivelpiece, A. W. 1967, PhFl, 10, 591

Scholer, M., Kucharek, H., \& Giacalone, J. 2000, JGR, 105, 285

Shukla, P. K., \& Stenflo, L. 1985, PhFl, 28, 1576

Skillman, S. W., O’Shea, B. W., Hallman, E. J., Burns, J. O., \& Norman, M. L. 2008, ApJ, 689, 1063

Spitkovsky, A. 2005, in AIP Conf. Proc. 801, Astrophysical Sources of High Energy Particles and Radiation, ed. T. Bulik, B. Rudak, \& G. Madejski (San Francisco, CA: ASP), 345

Trotta, D., \& Burgess, D. 2019, MNRAS, 482, 1154

Trotta, D., Valentini, F., Burgess, D., \& Servidio, S. 2020, arXiv:2011.10417 van Weeren, R. J., de Gasperin, F., Akamatsu, H., et al. 2019, SSRv, 215, 16 Vazza, F., Brunetti, G., \& Gheller, C. 2009, MNRAS, 395, 1333

Verscharen, D., \& Chandran, B. D. G. 2013, ApJ, 764, 88

Verscharen, D., \& Marsch, E. 2011, JPIPh, 77, 693

Winske, D., \& Quest, K. B. 1988, JGR, 93, 9681 